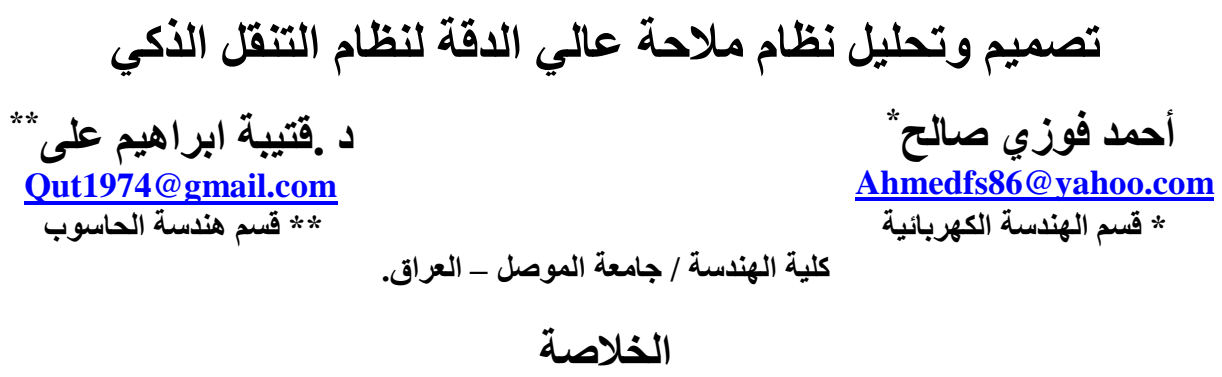

يقلم هذا البحث دراسة عن تصميم نظام جمع معلومات ومراقبة حركة المرور على شبكة الطرق في مدينة مثلا بناءً

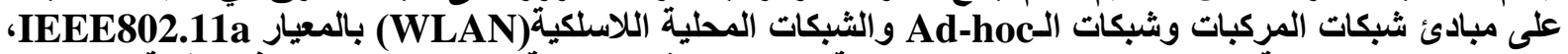

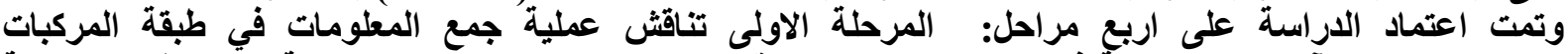

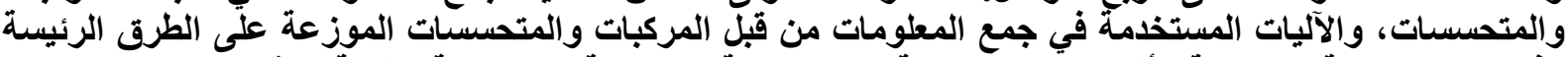

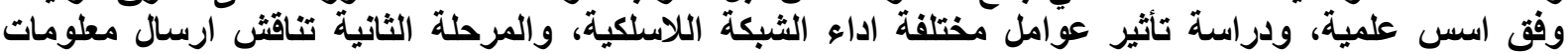

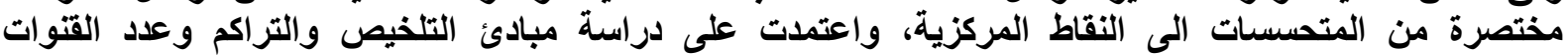

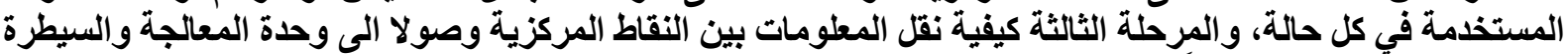

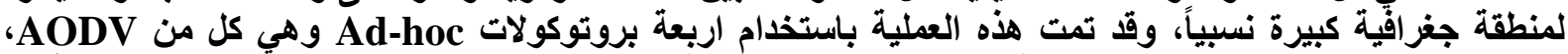

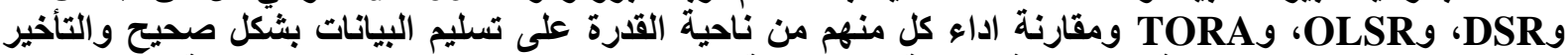

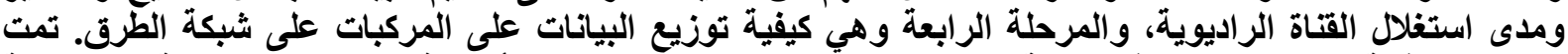

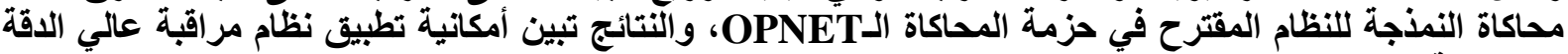
على شبكة ويزمن حقيقي.

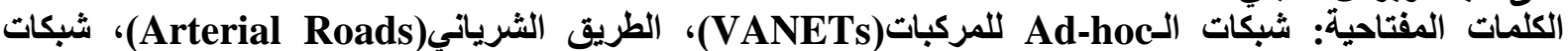

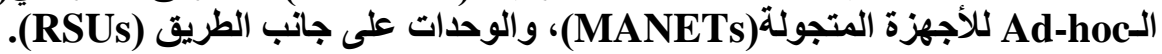

\title{
Design and Analysis of A High Resuluation Navigation System for The Intillegent Transportation System
}

\author{
Ahmed Fawzi Salih \\ * Dept. of Electrical Engineering** \\ Qutaiba I. Ali \\ Dept. of Computer Engineering \\ College of Engineering/ University of Mosul - IRAQ.
}

\begin{abstract}
This paper suggests an information gathering and monitoring system designed for vehicular adhoc networks(VANETs) and that implemented in a large area. The suggested infrastructure consists of low cost wireless sensors covering certain areas and connected to "the monitoring and control center" through a master node, the study is divided into four phases: the first phase discusses the information gathering process in vehicles and sensors level, the second phase discusses how to send the brief road traffic information to the master node. This process is based on the concepts of the summarization and aggregation and study the effect of using one or two channels for that purpose, the third phase focuses on the information transportation between the master nodes until it reaches to "the monitoring and control center", The mechanism of the information transportation in this phase is proposed to be in an ad-hoc manner using four adhoc protocols Ad-hoc On Demand Distance Vector Routing Protocol AODV, Dynamic Source routing DSR, Optimized Link State Routing Protocol OLSR and Temporally-Ordered Routing Algorithm Protocol TORA. The best protocol will be selected according to its data delivery, latency and average throughput on the radio channel and the fourth phase deals with dissemination of the gathered information to the vehicles. The proposed system is designed and simulated using "OPNET" package. The results indicate the ability of applying real time and high resolution monitoring system using these techniques.
\end{abstract}

$$
\text { قبل: } 2013 \text { - } 30
$$




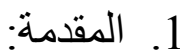

التطورات الحديثة في الثبكات المحلية اللاسلكية(Wireless Local Area Network WLAN) أدت الى ظهور نوع

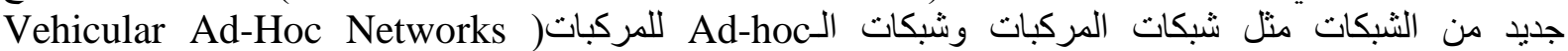

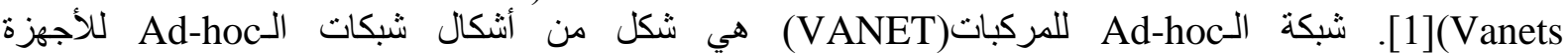
المتجولة) (Mobile Ad-Hoc Networks MANETs) وهي شبكات توزيعية ذاتية التنظيم[2]. شبكات المريكات المركبات

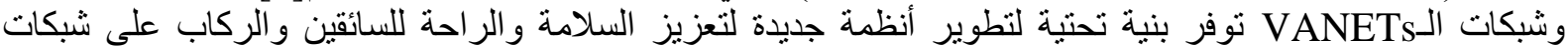

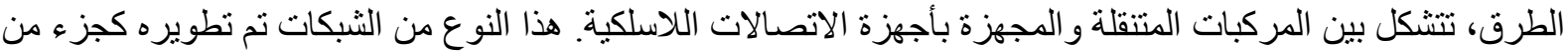

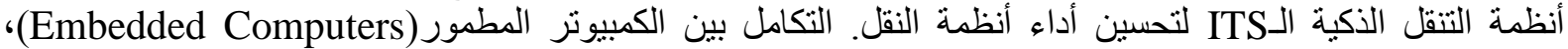

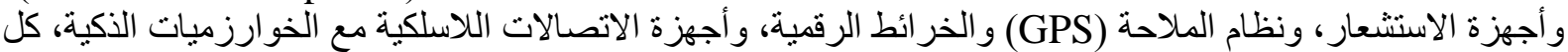

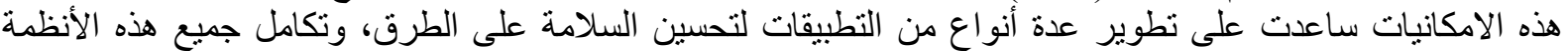

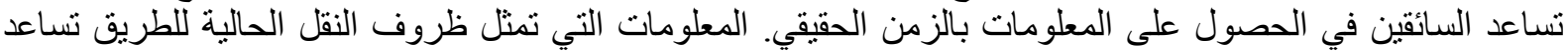

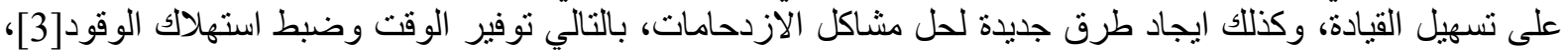

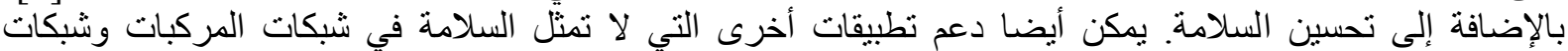

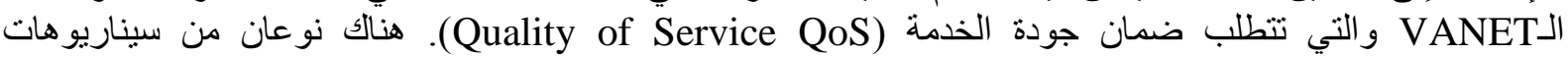
الاتصالات في شبكات المركبات وهي: اتصالات المركبات(Vehicle-to-Vehicle V2V) و اتصالات (لئات بين المركبات

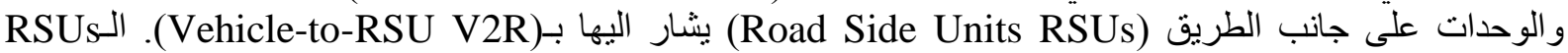
تتصل فيما بينها وكذللك يمكن ان تتصل مع الثبكات الاخرى مثل الانترنت. كما في النكل(1) [4] [5]. يتوقع ان توظف

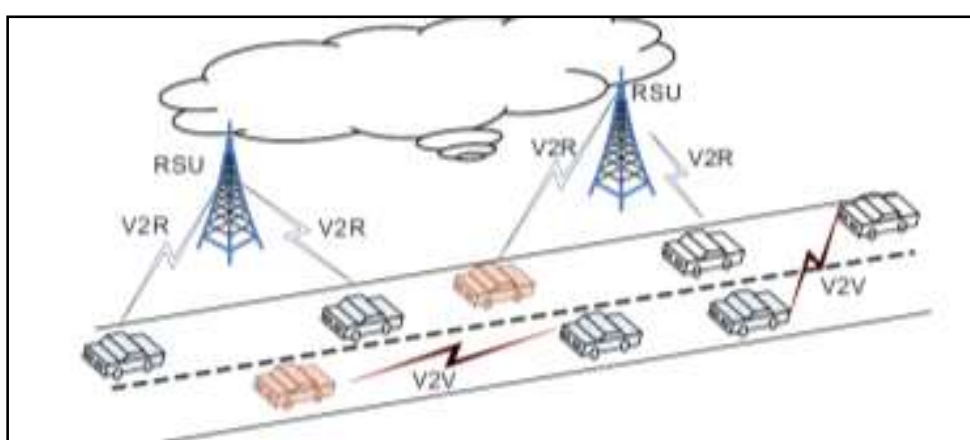

الثكل(1) يمثل تركيب شبكات المركبات.

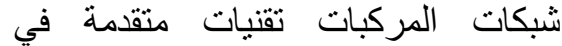

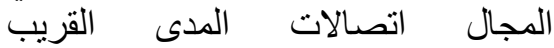
المخصصة (المال (Communications DSRC

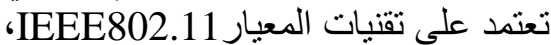
و التي تكون ملائمة لبيئة شبكات المركبات

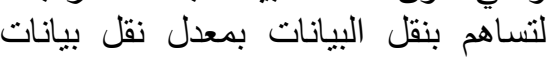
عالي في البيئات التي تخضي لتصع لعمليات تغنير مستمرة مثلا الاتصالات في شيكات فيكات المركبات والتي تتطلب معدلات نقل فئل عالية[6].

2- ألأعمال السابقة هنالك الكثير من الجهول الإلبود التي بذلت في اقتراح تصنيفات جديدة لجمع المعلومات ونشرها، و التقنيات المستخدمة و الخو ارزميات المتبعة لإنتاج معلومات مفيدة لانظمة ملاحة المركبات. و فيما يأتي بعض لهن من هذه الجهود:

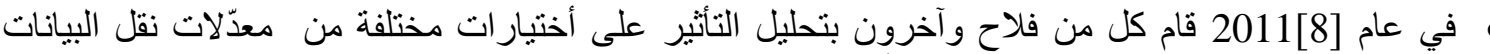

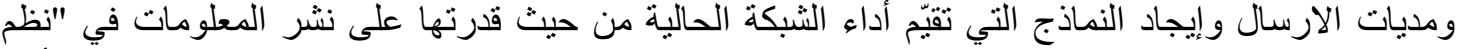

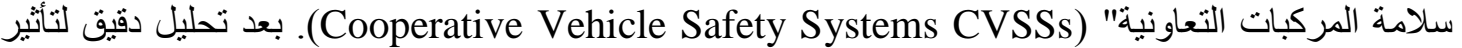

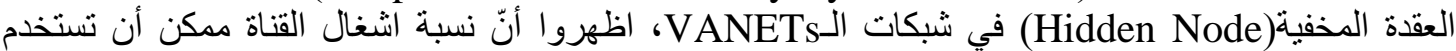
كمقدار لنجاح اتتشار المعلومات، وبالتنالي، تحدد مدى نجاح نظام Cooperative Vehicle Safety Systems

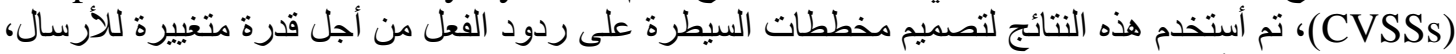

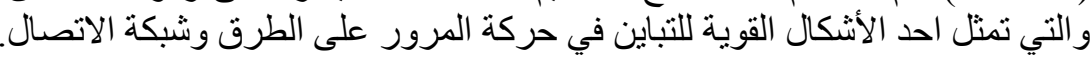

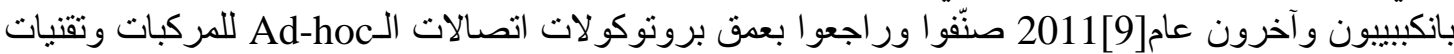

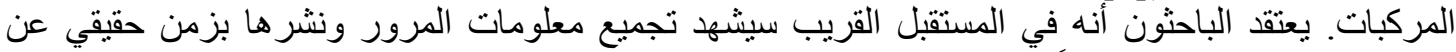

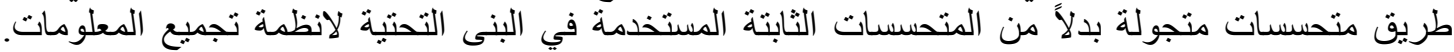

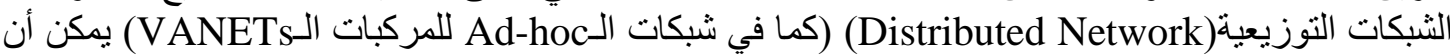

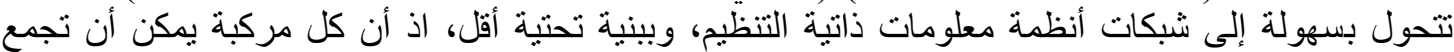

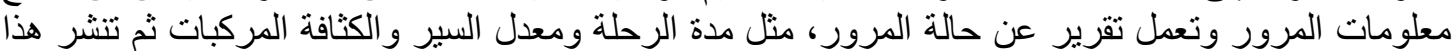
التقرير. 
قام كل من عالم وآخرون عام 2012[10] بمناقشة معلومات المو اقع (Position Information) التي هي شرط التمات

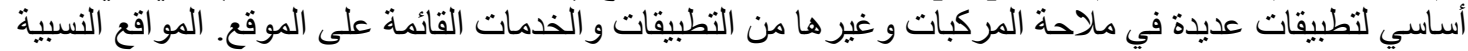

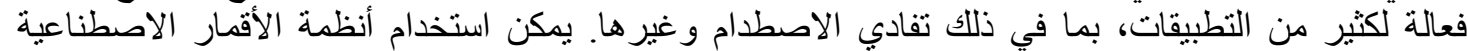

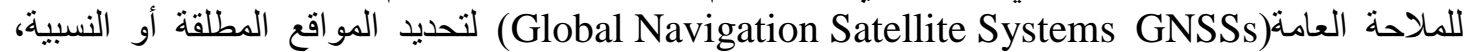
ولكن مستوى الدقة لا تفي بمنطلبات العديد من التطبيقات، لهذا نم إستخدام تقنية التموقع التعاوني( Cooperative (Positioning CP

الـ

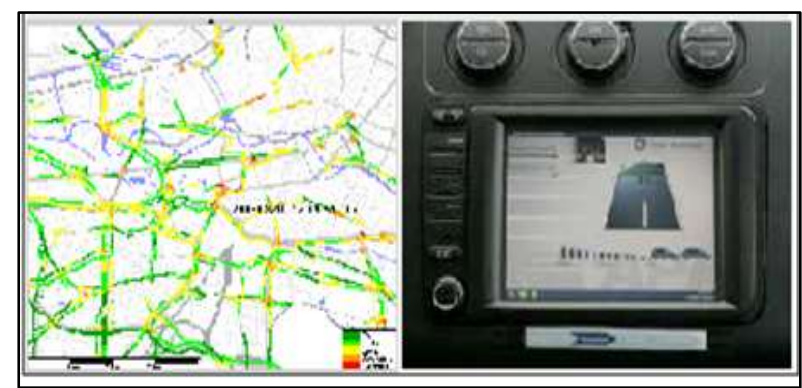

الثكل(2) يوضح أنموذج لواجهة نظام ملاحة.

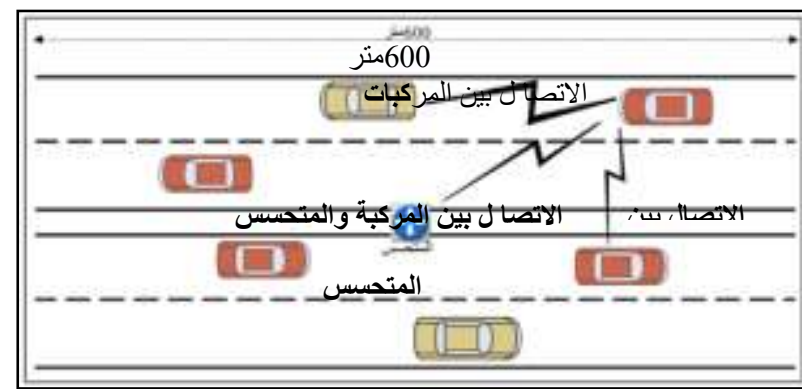

شكل(3) البنا الاساسي للنظام والمشهز المحلي للملاحة.

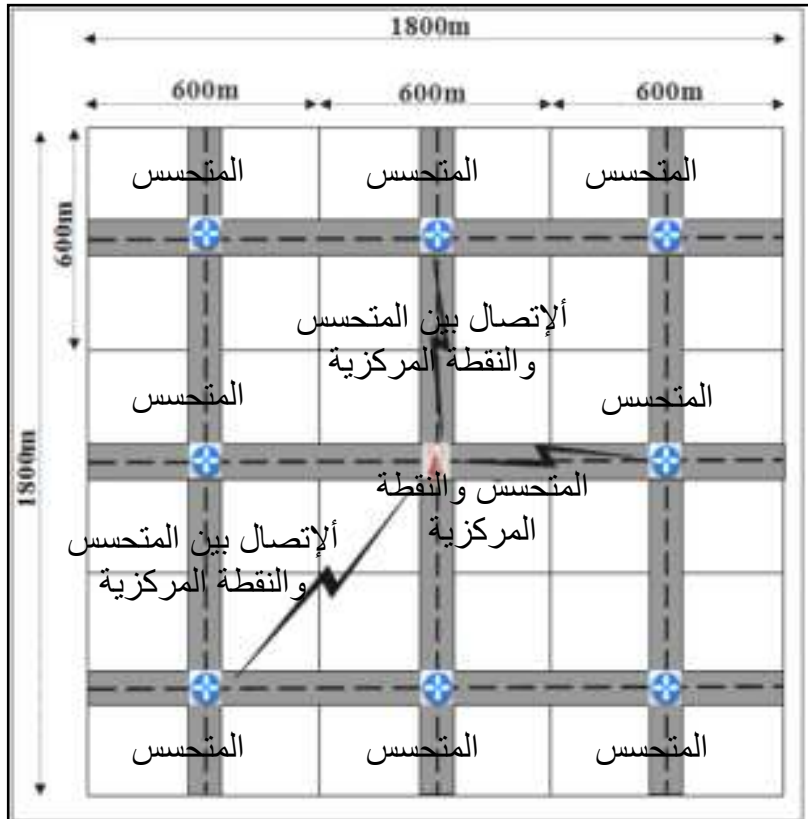

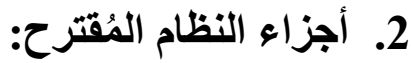
والمركبات: المركبة لكي تستطيع نكوين نظام المام ملاحة

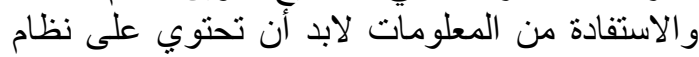

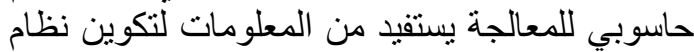

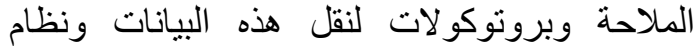

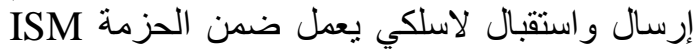

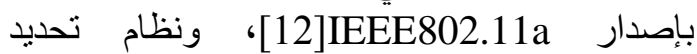

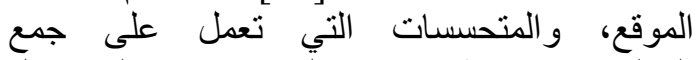

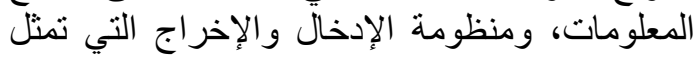
الواجهة للتفاعل بين المستخدم والإلظام والخراج وكما في تئي

ب- المتحسسات: تنتشر في الطرق الرئيسة لتجمع المعات

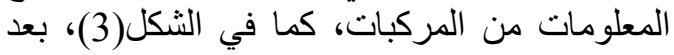

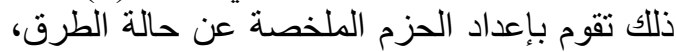

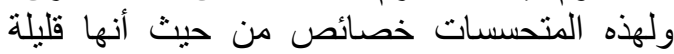

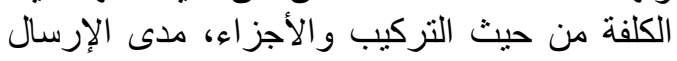

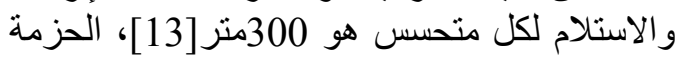
التي تعمل عليها ضمن الحزمة GHz5 بـإيندار

.IEEE802.11a

ت- النقاط المركزية: يكون للنقطة مركزية على الاكثر

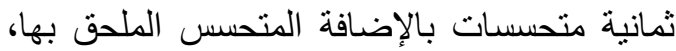

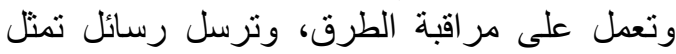

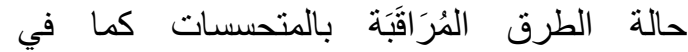
الثكل(4). النقاط المركزية المبنة المنتشرة ترسل المعلومات الى مركز المر اقبة السبطرة.

ثـ مركز السبطرة والمراقبة: وهو الخادم الاساسي ويعمل على تجميع المعلومات من النقاط المركزية،

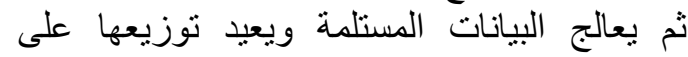

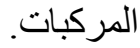

الثكل(4): النقطة المركزية مع المتحسسات الملحقة بها. 


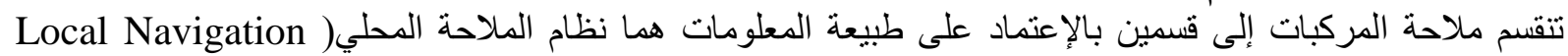

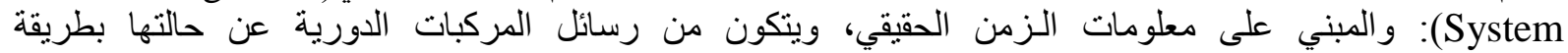

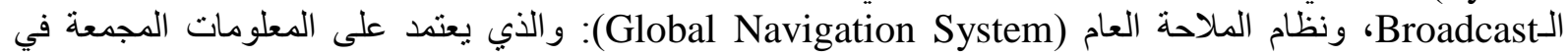

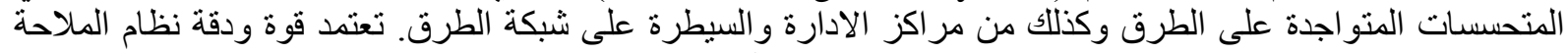

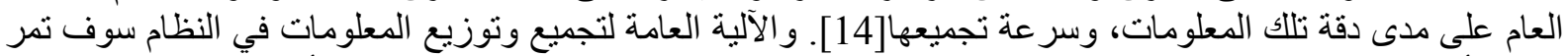

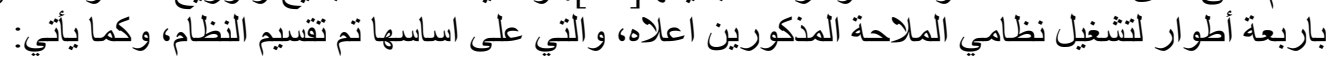

الطور الاول: هو اساس نظام الملاحة المحلي، ويعتمد على الرسالة دورية من المركبات(نبضات القلب أو المنارة)، والتي التي

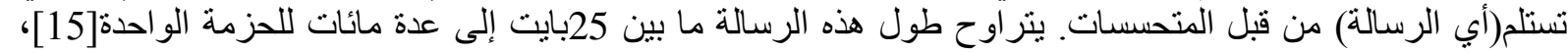

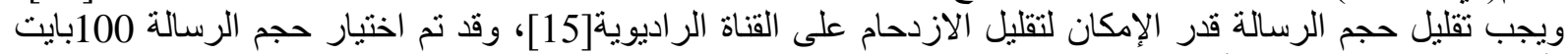

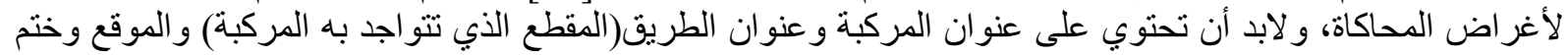

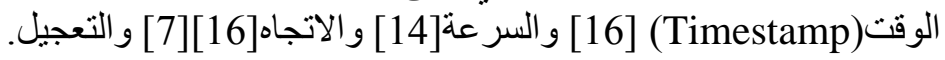

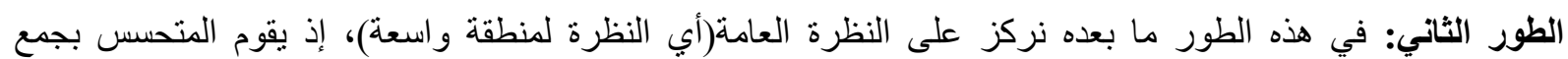

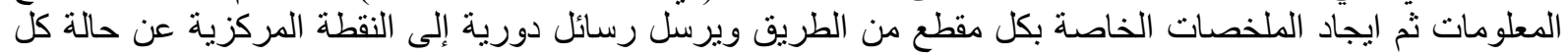

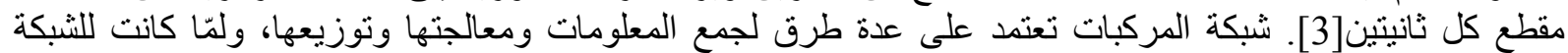

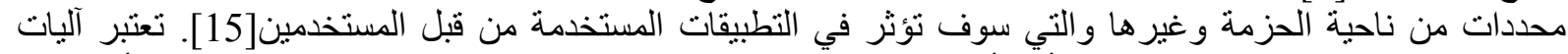

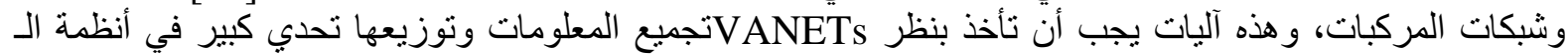

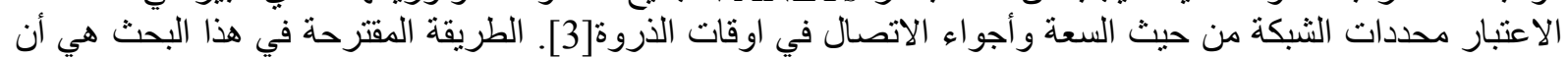

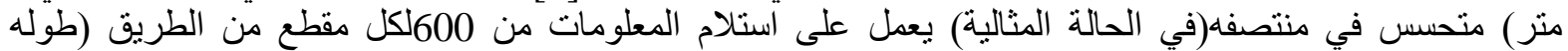

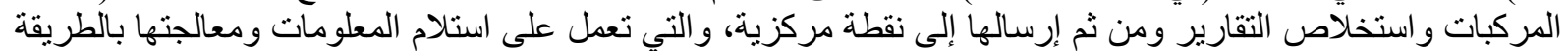

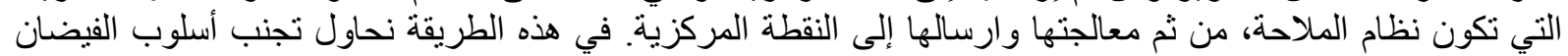

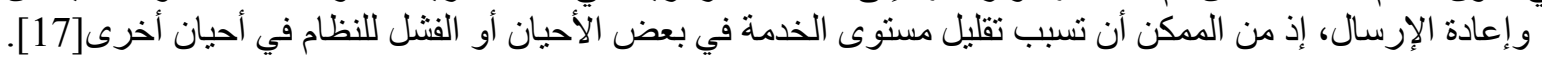

الطور الثالث: تقوم النقطة المركزية بمعالجة هذه الرسائل ثم تكوّن رسالة عن المتحسسات التي تجمع المعلومات منهم

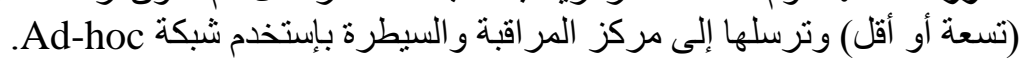

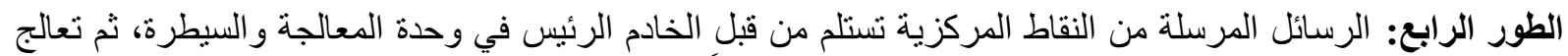
ويعاد توزيعها لتكوين نظام الملاحة العامة (لمنطقة كبيرة(مدينة مثلاً)). ولم يتم التطرق التئل إلى هذا الطور في هذا البحث.

\section{4. دراسة النظام المقترح بأسلوب المحاكاة:}

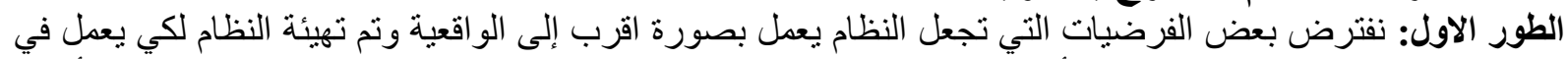

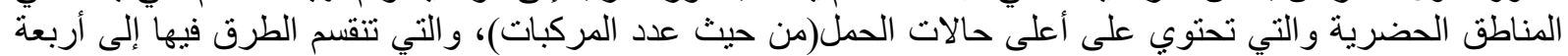

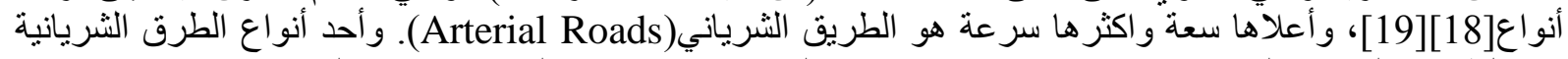

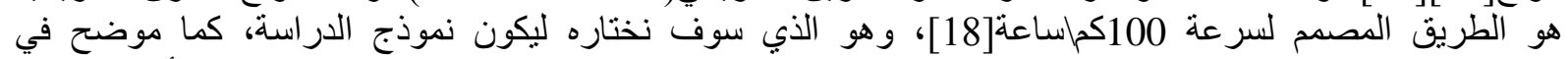

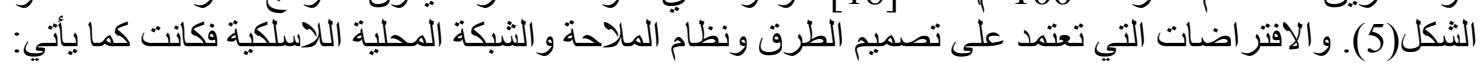

$$
\begin{aligned}
& \text { 1) تصميم الطريق لسر عة 100كم|ساعة [18]. }
\end{aligned}
$$

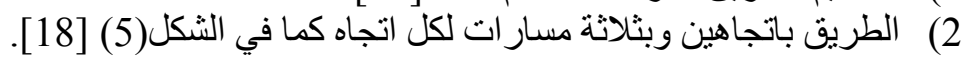

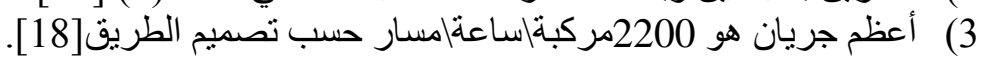

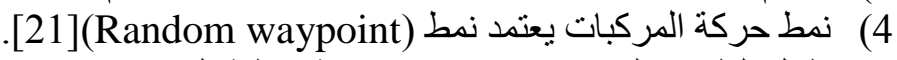

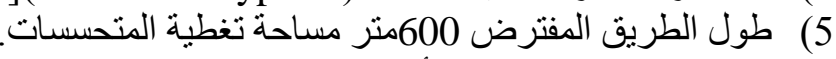

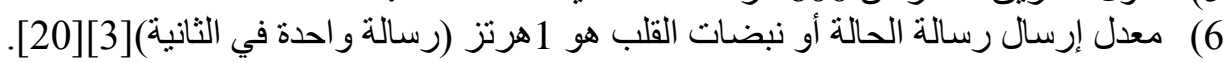

$$
\begin{aligned}
& \text { 7) طول الرسالة هو 100بايت. }
\end{aligned}
$$

حسب معايير التصميم فأن المركبات عندما تسير بسرعة 100كم|ساعة على الطرق المصممة لسرعة 100كم|ساعة فهذا يعني أفضل مستوى خدمة (Level Of Service LOS) قد قدمته هذه الطرق مع معند معدل 
الجريان(Flow Rate) يقدر بـ2200 مركبة|مسار اساعة و الذي يتيح(أي معدل الجريان) أمكانية تحديد عدد المركبات في إسيات

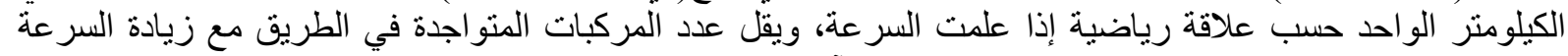
ويزداد كلما قلت السرعة للمركبات [18]. حسب العباف العلاقة الآتية:

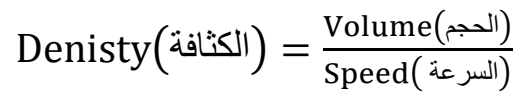

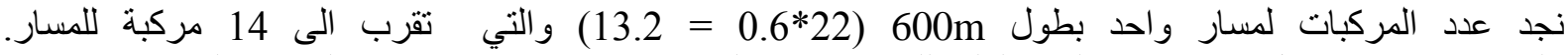

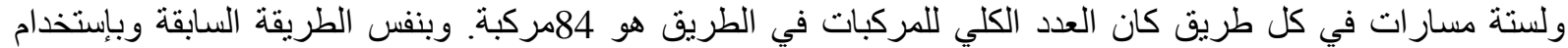

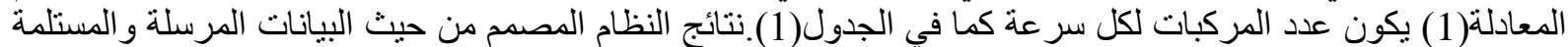

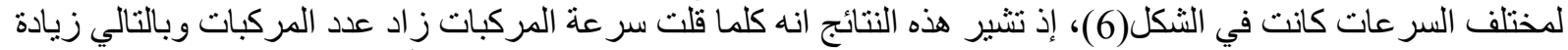

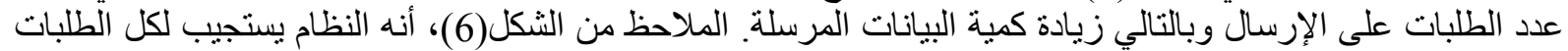

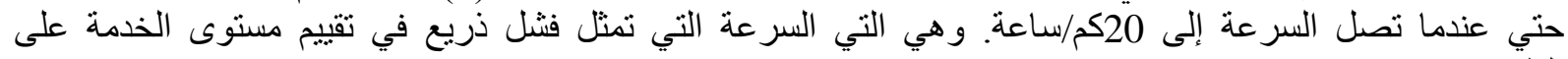

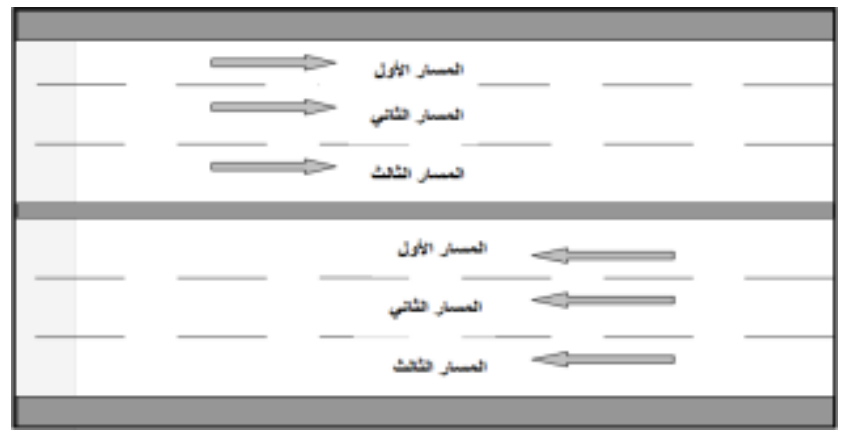

الجدول(1) عدد المركبات لكل مسار حسب السرع (عدات

الشكل(5) يوضح انموذج الطريق الشرياني.

\begin{tabular}{|c|c|c|}
\hline معدل السر عة & عدار المركبات & |طريق المركبات \\
\hline 100كم|ساعة & 14 & 84 \\
\hline 80 كم|ساعة & 17 & 107 \\
\hline 60 كملساعة & 22 & 132 \\
\hline 40 كم|ساعة & 33 & 198 \\
\hline 20 كم|ساعة & 66 & 396 \\
\hline
\end{tabular}

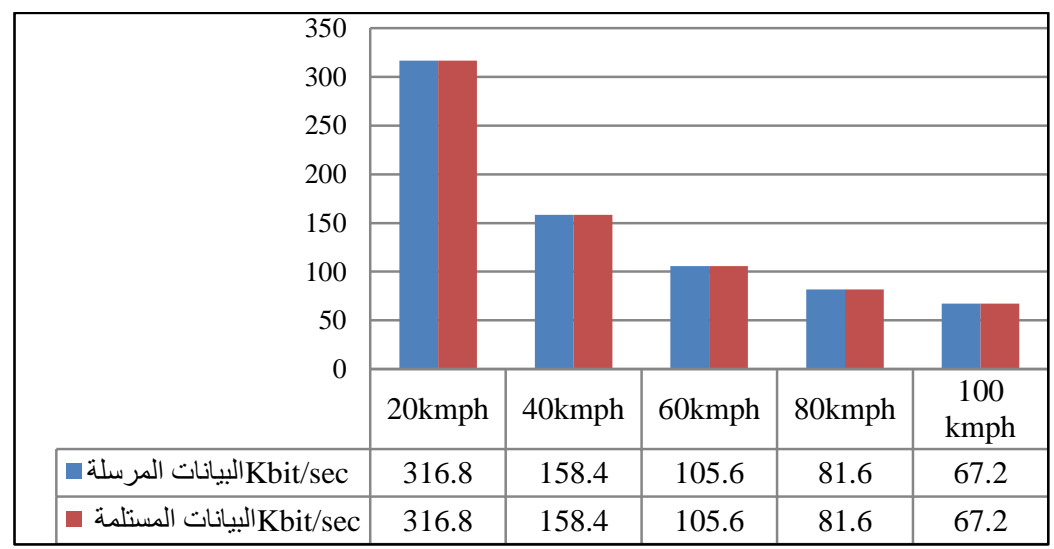

الثكل(6) يوضح البيانات المرسلة و المستلمة عند المتحسس للسر عات (20,40,60,80,100) كم|ساعة.

الطور الثاني: المتحسسات تستلم المعلومات من المركبات وتقوم بمعالجتها، ومن ثم إرسالها إلى بنظام الارسال الاحادي

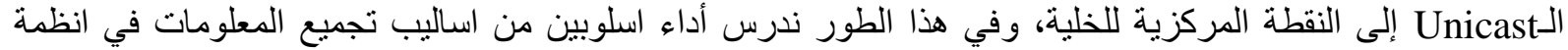

المركبات و هي التلخيص(Summarization) و التر اكم(Aggregation).

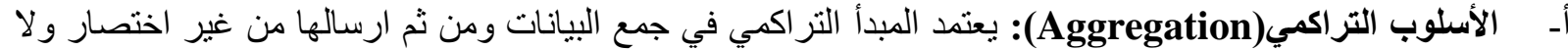

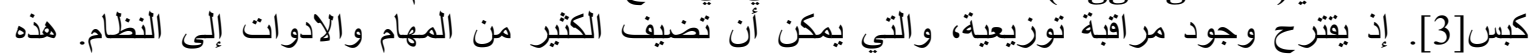




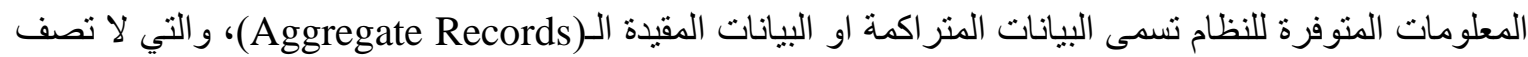

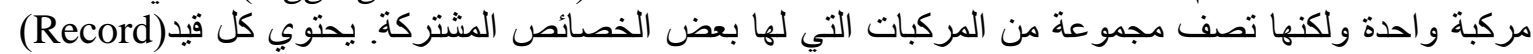

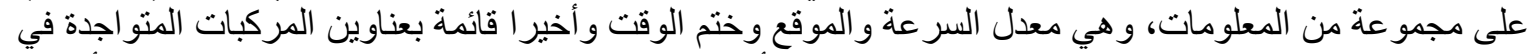

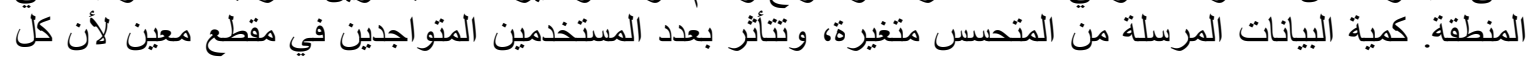

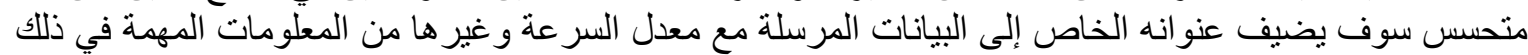

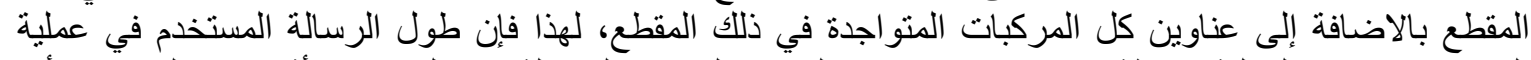

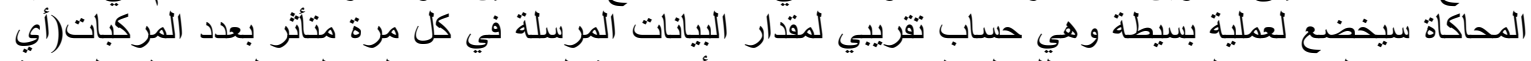

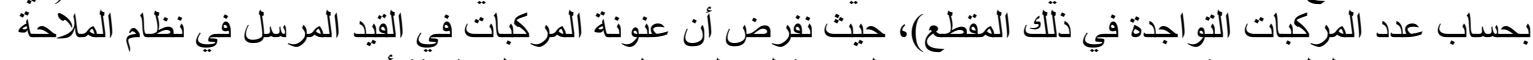

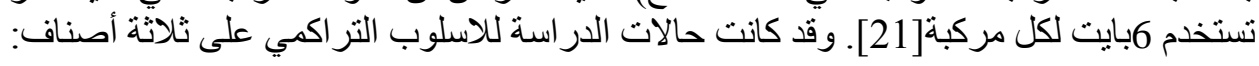

1. الحالة الأولى(الحالة المثالية): هذه الحالة لفحص أفضل أداء ممكن أن بحصل للشبكة من دون أي تأثثر من الاجزاء ألثاء

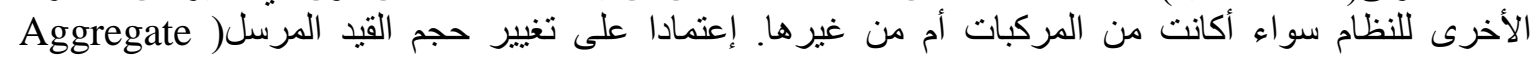
(Records

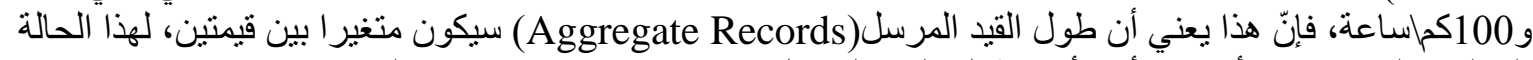

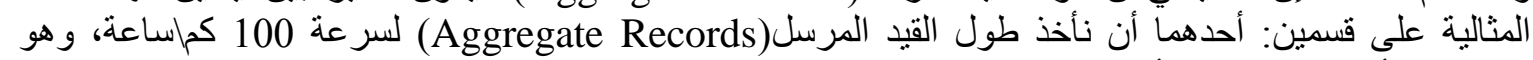

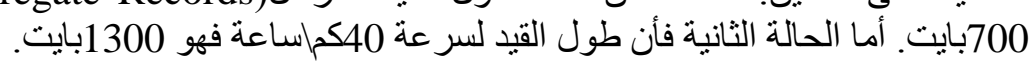

2. الحالة الثانية: النظام في هذه الحالة يعمل مع تو اجد المركبات على الطرق بسر عة 100كملساعاعة. عدد المركبات يكون

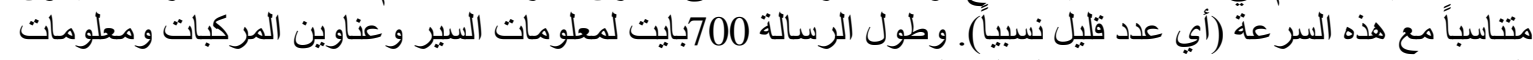
السيطرة والإدارة، وقد تم دارسة هذه الحالة على نمطين هما:
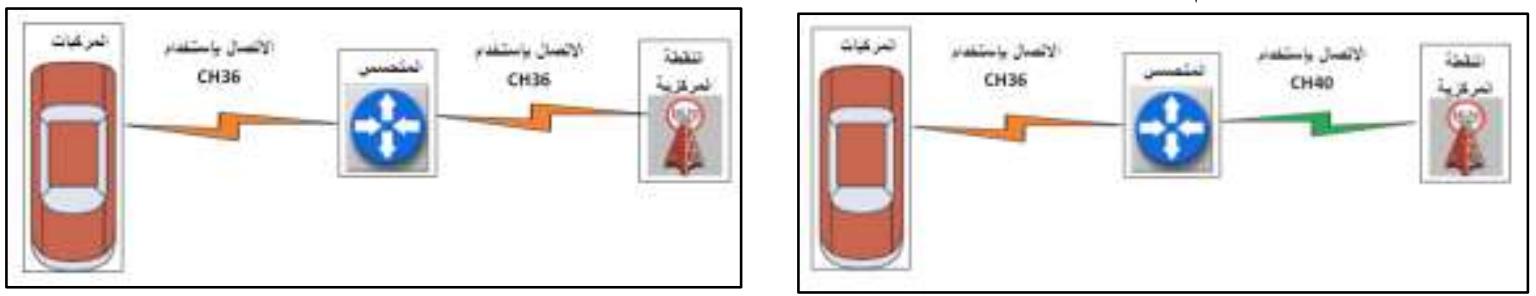

الشكل(7A) الجزء يوضح الاتصال بإستخدام قناة واحدة. الشكل(7B) يوضح الاتصسال بإستخدام

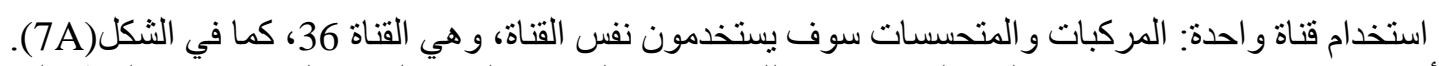

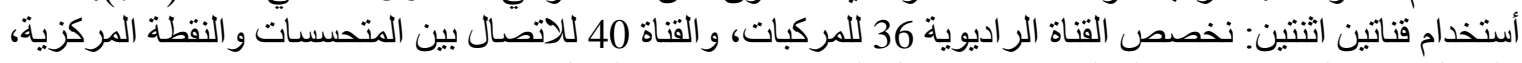

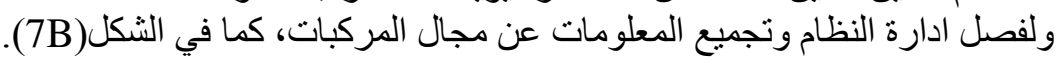

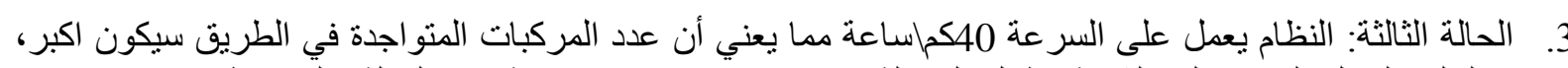

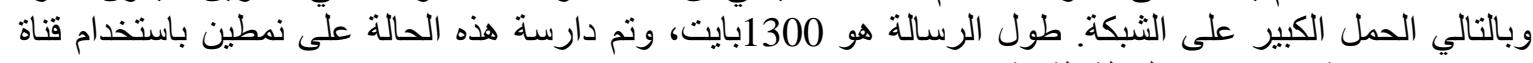
واحدة وقناتين اثنتين كما في الحالة الثانية.

النتائج للاسلوب التراكمي في الثكلين(8) و(9)، تثير إلى أنّه عندما تكون الاجواء مثالية(كما في الحالة الاولى)

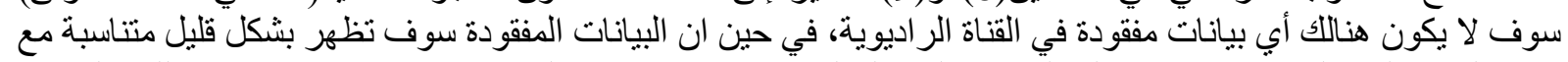

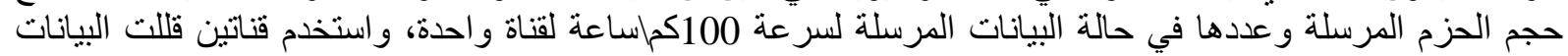

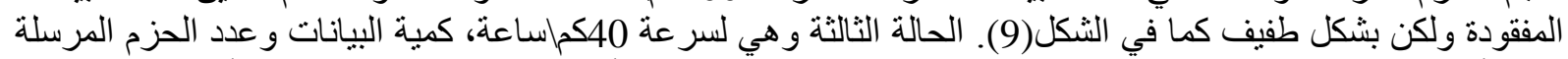

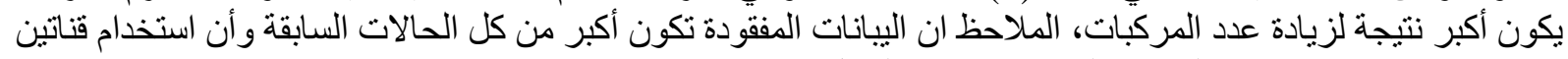

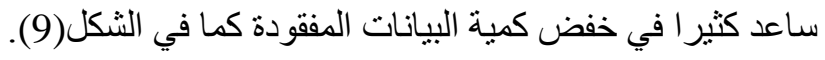


علي: تصميم وتحليل نظام ملاحة عالي الدقة لنظام التنقل الذكي

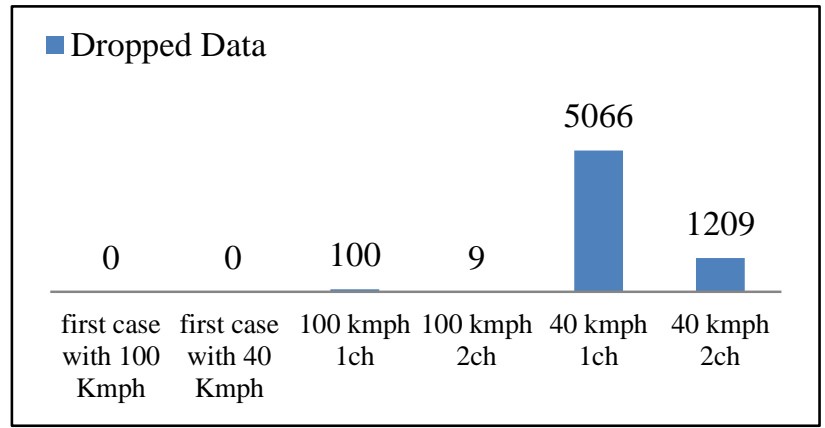

الثكل(9) معدل البيانات المفقودة لكل حالات الدر اسة في نظام الـTrafficview n Average Load Kbps

2892.82892 .8

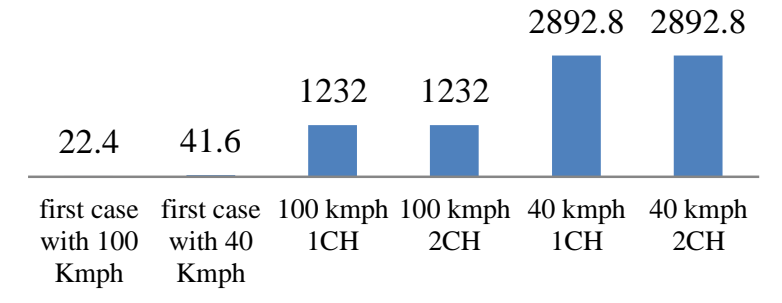

الثكل(8) يوضح معدل الحمل (Average Load)على

الثبكة في نظام الـTrafficview

بـ ـ اسلوب التلخيص (Summarization): هنالك الكثير من الخوارزميات التي تعتمد على مبدأ التلخيص منها(-Self) S22](Organizing Traffic Information System SOTIS

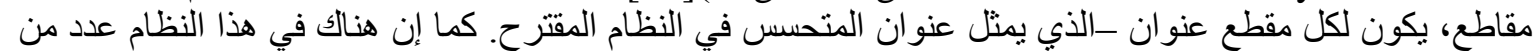

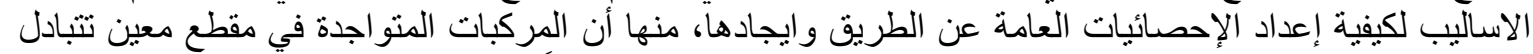

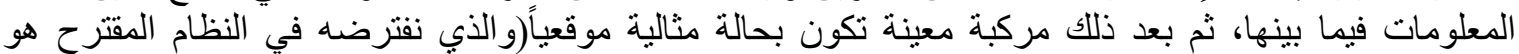

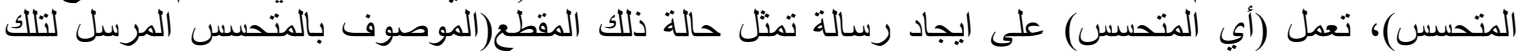

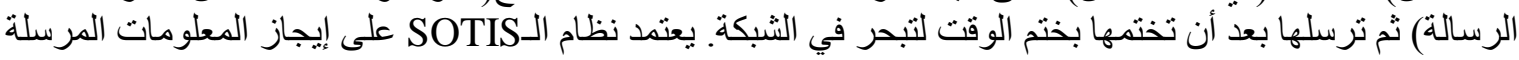

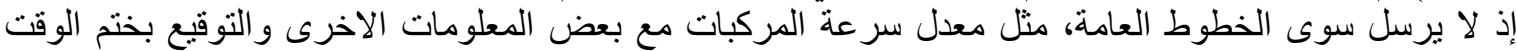

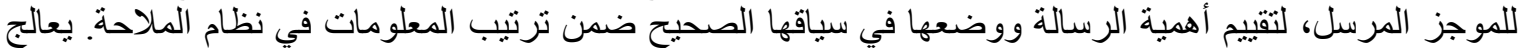

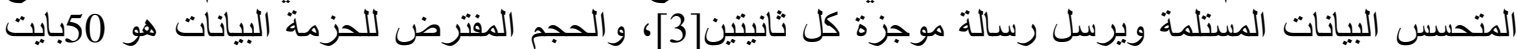

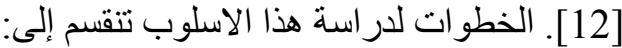

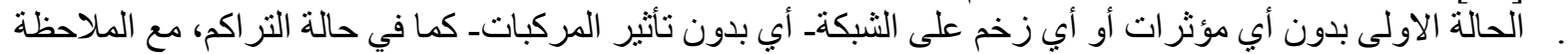

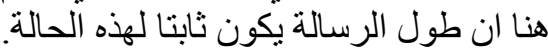

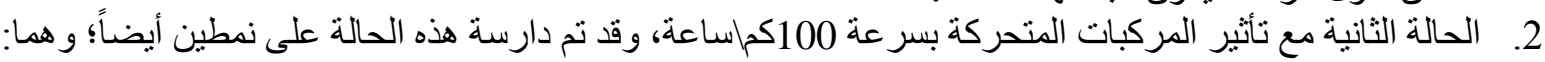

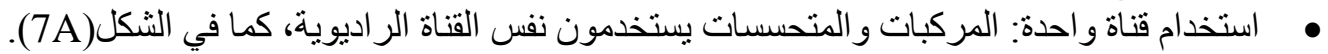

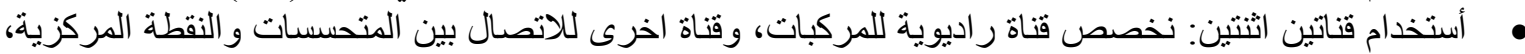

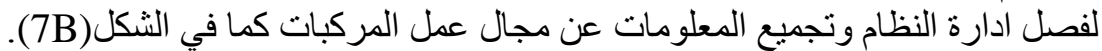

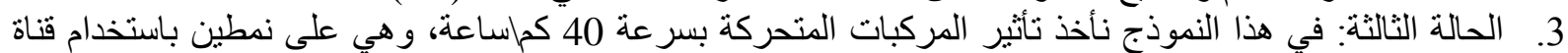
واحدة وقناتين اثثتين كما في الحالة الثانية.

الثكل(11) يبين أنه لا يوجد بيانات المفقودة في الحالة المثالية، في حين أن البيانات المفقودة كانت عدة بايتات عند الئد

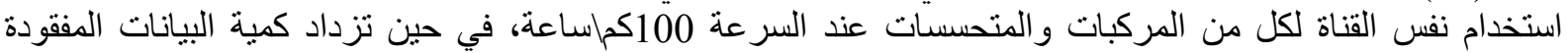

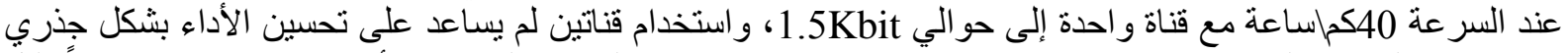

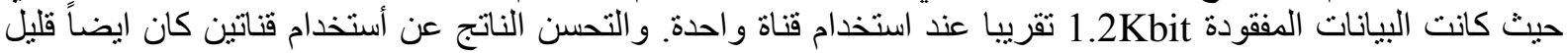

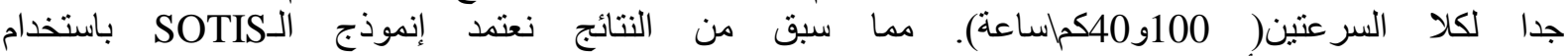
قناة واحدة، كونه افضل أداءً من الـTrafficview، و استخدام قناتين كان ذو فائدة محدودة ولم يقضِ على البيانات المفقودة.

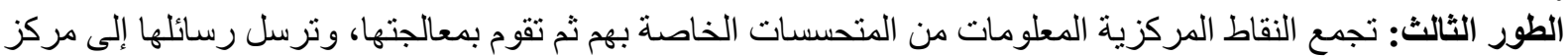

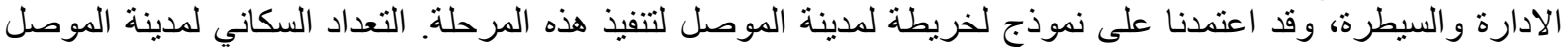

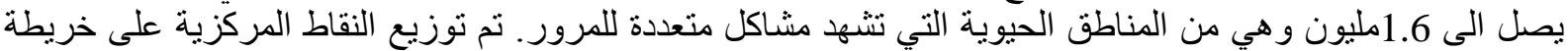

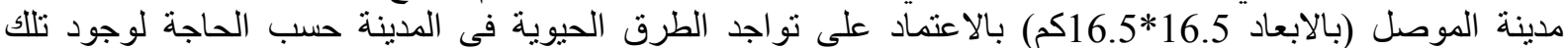

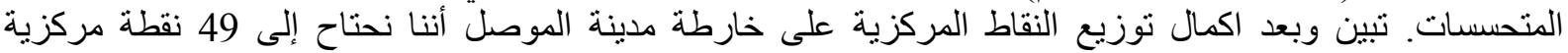

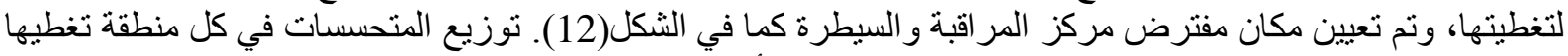

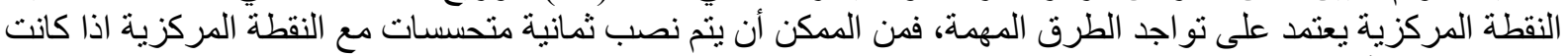

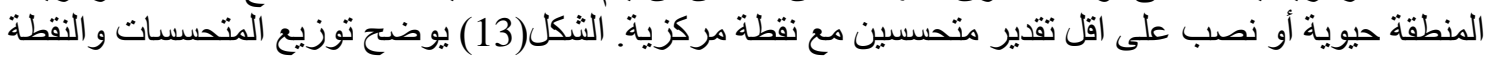




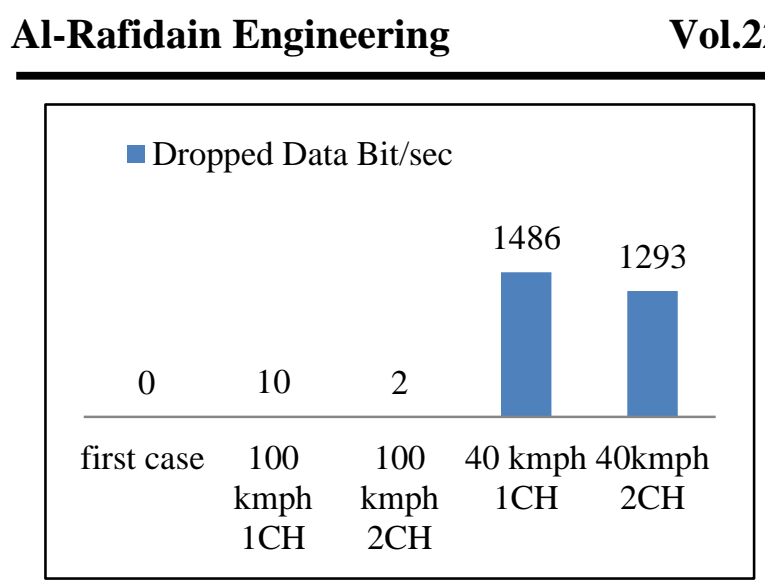

الشكل(11) معدل البيانات المفقودة لحالات الدراسة في الـSOTIS
No. 4

May 2014

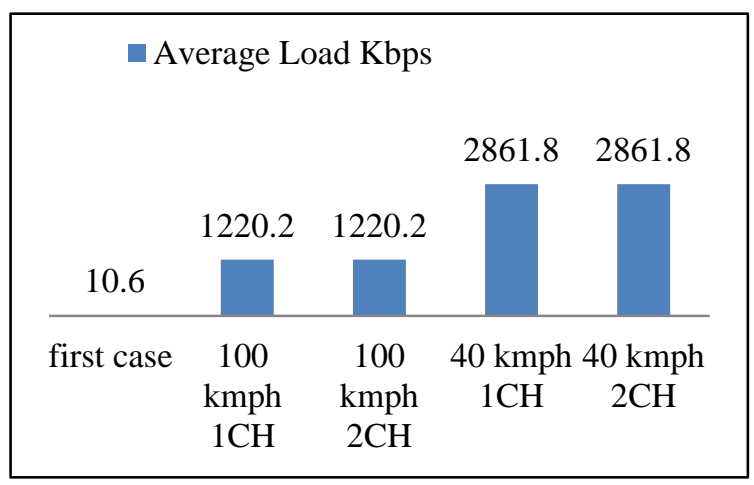

(10) الثكل (10) معدل الحمل (Average Load)

لحالات الدر اسة في SOTIS

المركزية على منطقة معينة. نم انشاء شبكة Ad-hoc لدراسة أفضل أداء للشبكة اللاسلكية باعتماد الخصائص المناسية

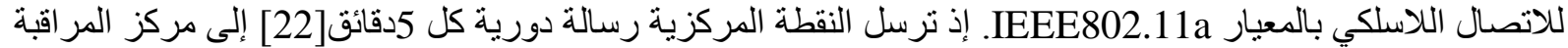

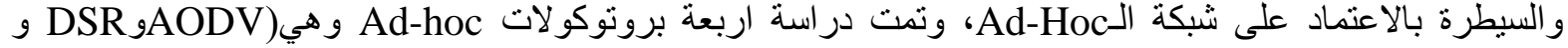
(TORAو OLSR

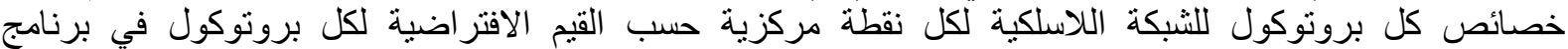

الـINET

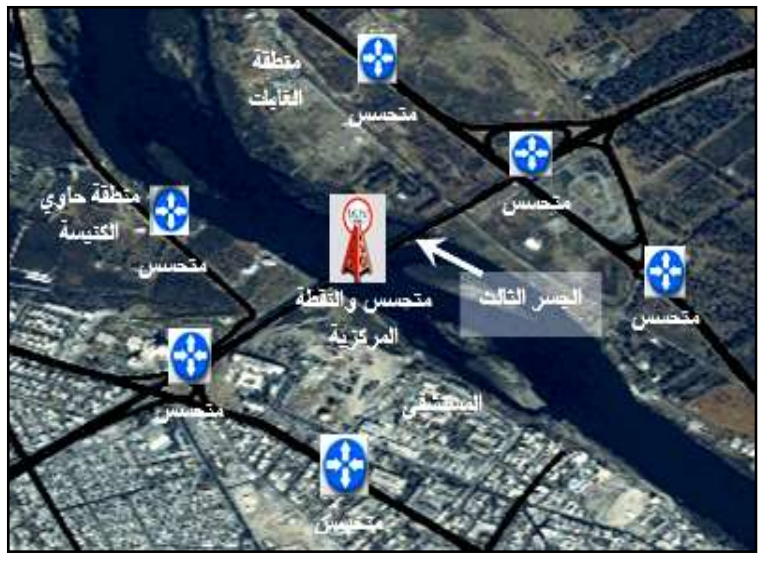

الثكل(13) انموذج للنقطة المركزية مع المتحسسات.

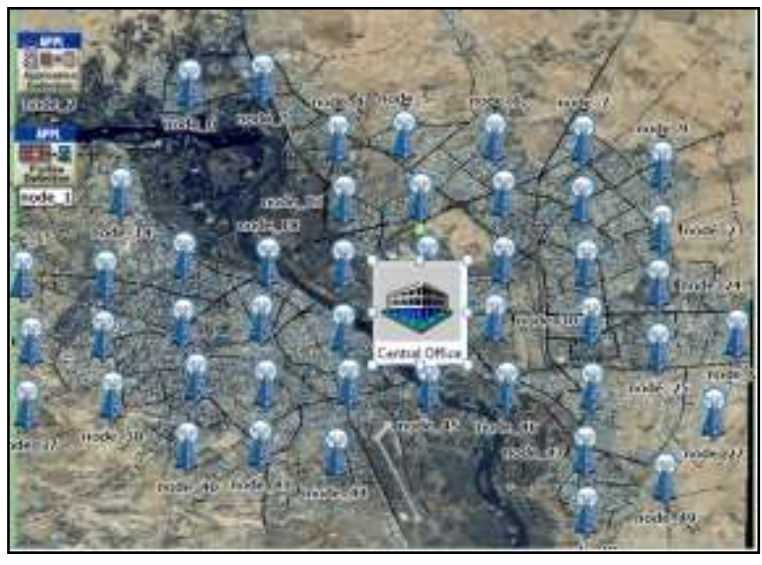

الثكل(12) توزيع النقاط المركزية في مدينة الموصل.

وقد أعتمدت عدة قياسات في هذا الجزء من البحث و هي كما يأتي[13]:

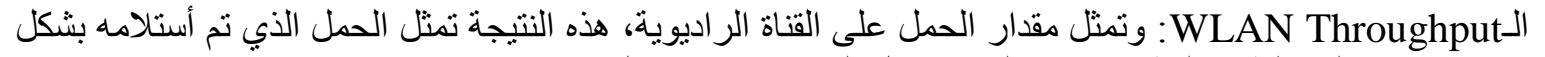

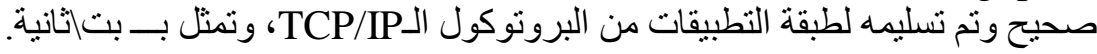

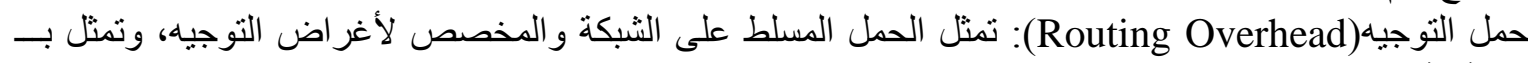
بتانثانية.

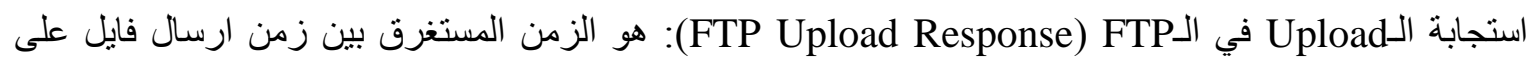

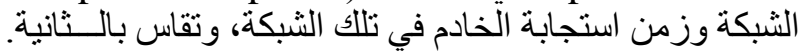
أما نتائج عملية المحاكاة فكانت كما يلئي:

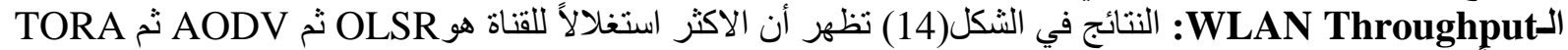

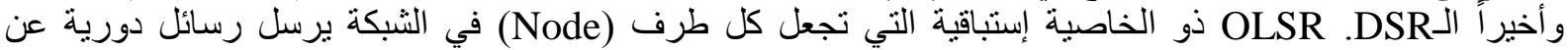

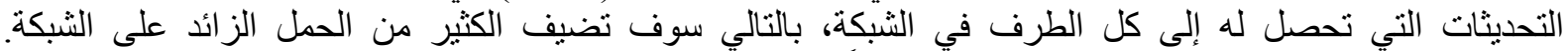

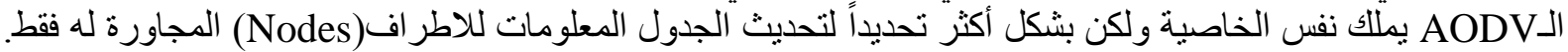

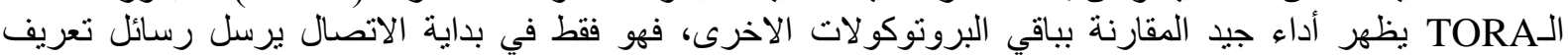

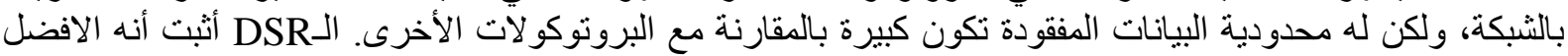


لتطبيقنا لأسباب، منها أنه بروتوكول تفاعلي و المعلومات المنوفرة من أي اتصال سابق تدعم الاتصالات الجديدة لتوفير

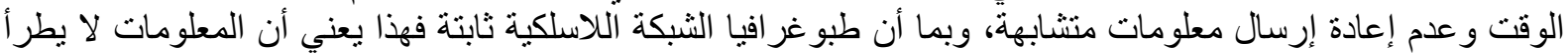

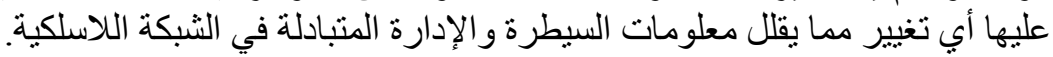

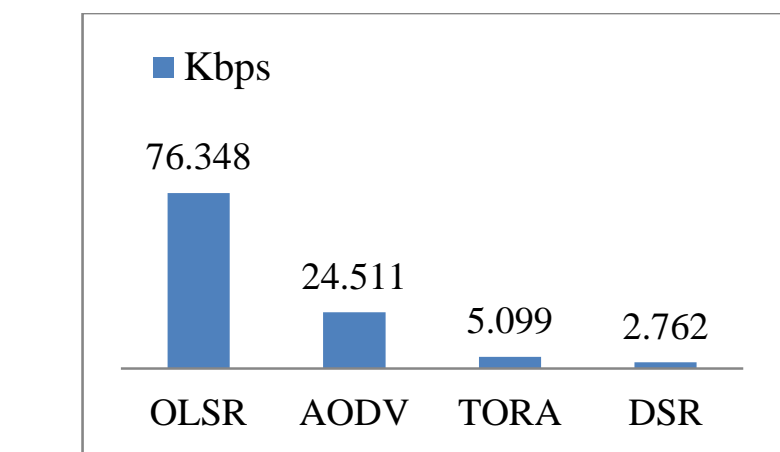

النكل(15) يوضح البيانات التوجيه المتبادلة في

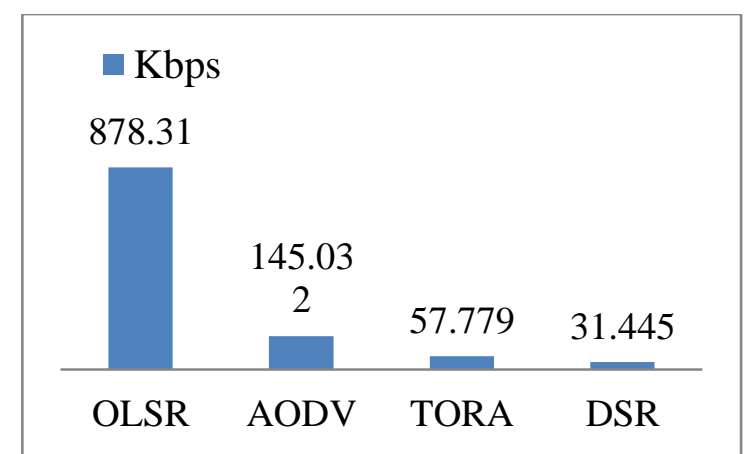

الشكل(14) بوضح الـLAN Throughput بروتوكو لات لأربعة

حمل التوجيه(Routing overhead): الشكل(15) يمثل الحمل المسلط على الثبكة اللاسلكية الناتج عن عملية التوجيه

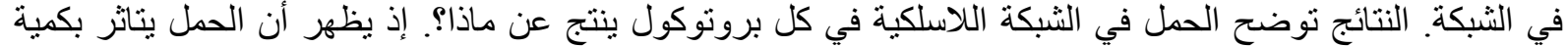

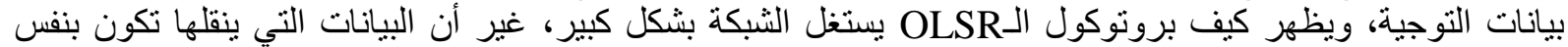

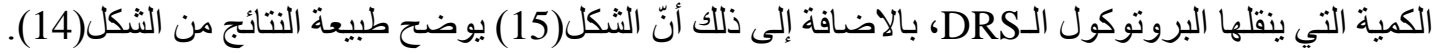

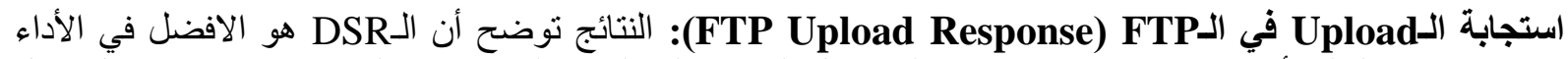

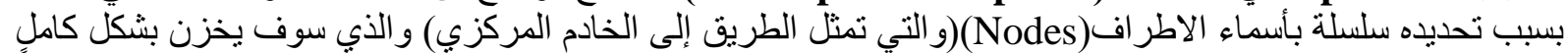

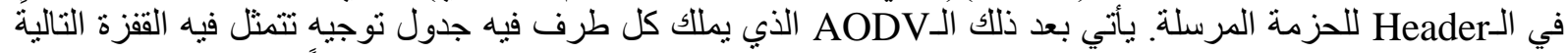

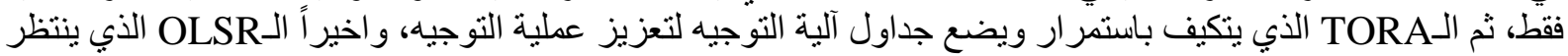

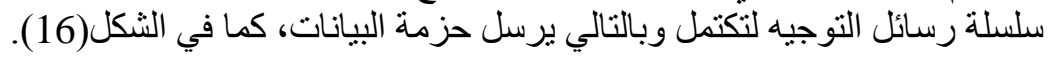

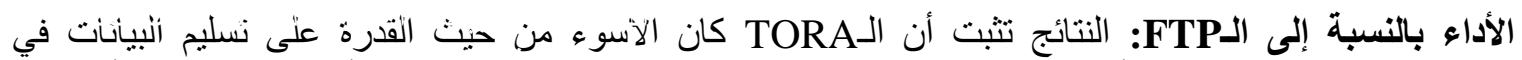
بروتوكول الـFTP و هذا يرجع إلى أسباب منها كون عدد الاطر اف(ivodes) في النثبكة ممكن أن يكون العامل الأبرز في في أداء النظام. في حين أن الـDSR يتميز بالقدرة العالية على تسلم البيانات بشكل صحيح كما في الثكل(17).

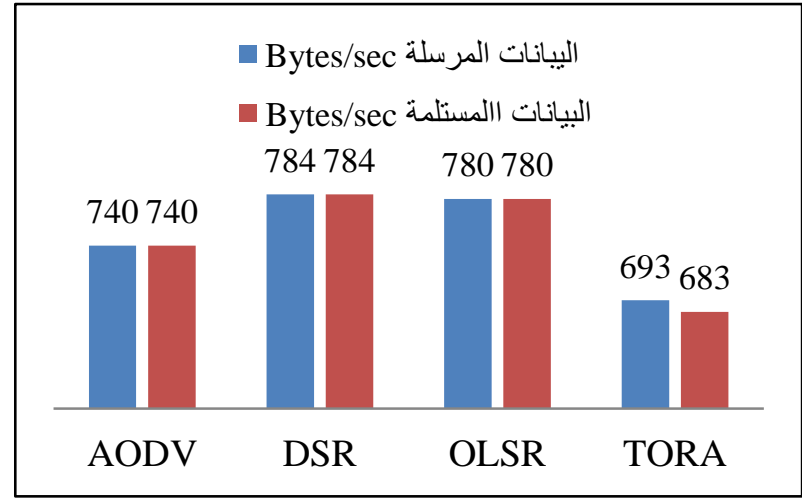

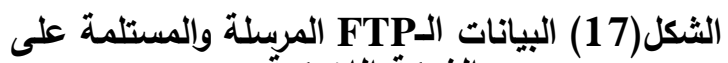
الثبكة الللاسكية.

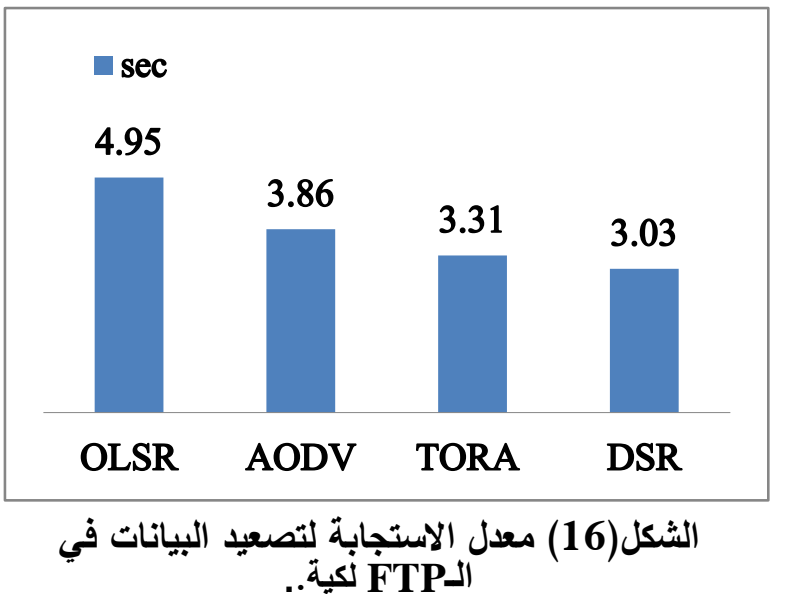

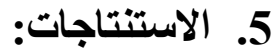




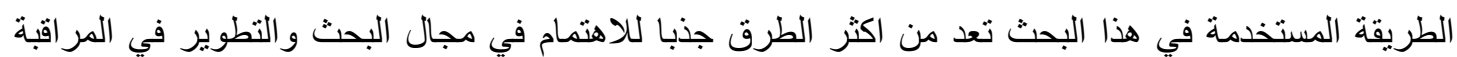

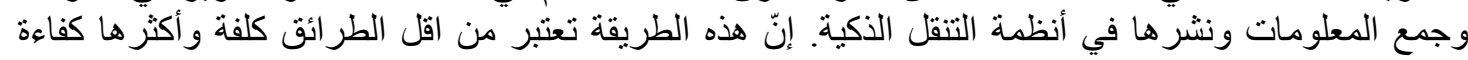

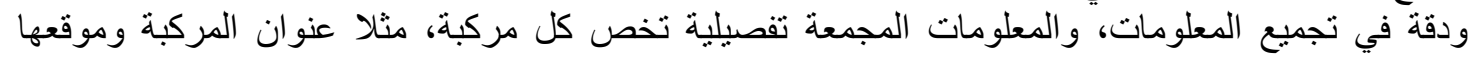

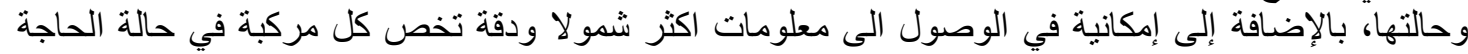

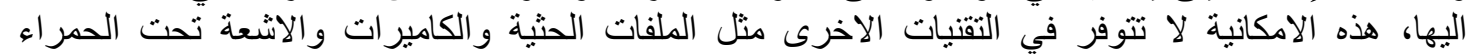

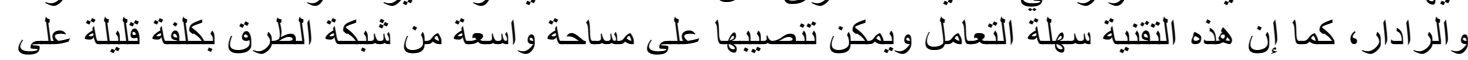

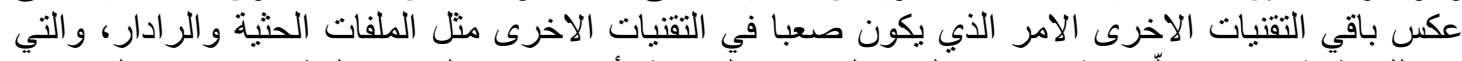

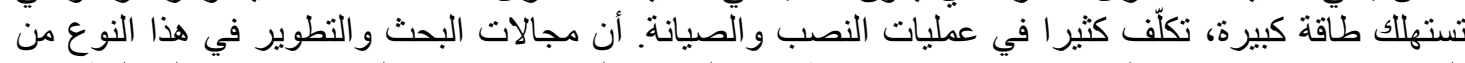

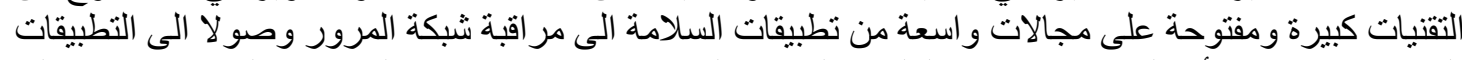

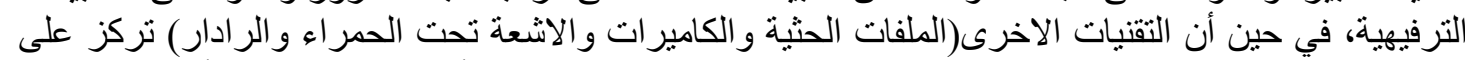

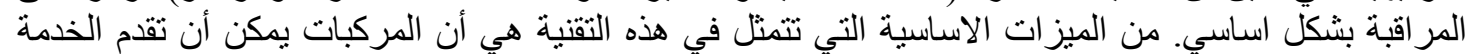

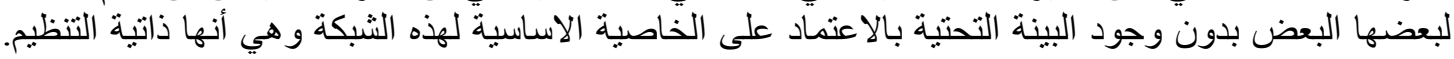

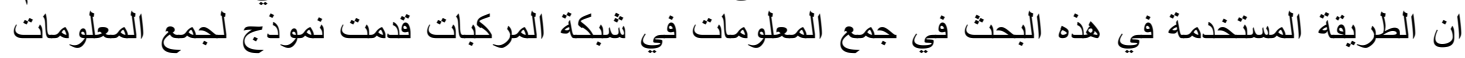

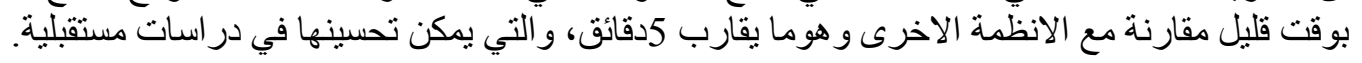

(المصادر:

[1] T. Sivaharan, G. Blair.and G. Coulson, "GREEN: A Configurable and Re-configurable Publish-Subscribe Middleware for Pervasive Computing”, PP 732-749, OTM Confederated International Conferences, CoopIS, DOA, and ODBASE, Springer-Verlag Berlin Heidelberg, 2005.

[2] M. Fiore, J. H"arri, F. Filali and C. Bonnet, "Vehicular Mobility Simulation for VANETs",IEEE Simulation Symposium, 2007. ANSS '07, 40th Annual, PP301 - 309, March 2007.

[3] T. Nadeem, S. Dashtinezhad, C. Liao and L. Iftode, "TrafficView: Traffic Data Dissemination using Car-to-Car Communication", ACM Mobile Computing and Communications Review (MC2R), Vol. 8, No. 3, PP. 6-19, July 2004.

[4] X. Wang, "Mobile Ad-Hoc Networks: APPlications", InTech, Janeza Trdine 9, 51000 Rijeka, Croatia, Volume 6, PP 307-321, 2011.

[5] J. Toutouh and E. Alba, "Performance Analysis of Optimized VANET Protocols in Real World Tests", IEEE Wireless Communications and Mobile Computing Conference (IWCMC), 2011 7th International, PP1244 - 1249, 2011.

[6] W.Wang, F. Xie and M. Chatterjee, "An Integrated Study on Mobility Models and Scalable Routing Protocols in VANETs”, 2007 Mobile Networking for Vehicular Environments, PP97 - 102, 2007.

[7] Y. Wu, Z. Shao, W. Li, L. Shen and X. Li, "A Novel Design and Realization of the Vehicular Driving Navigation System based on VANET", IEEE Proceedings of the 8th World Congress on Intelligent Control and Automation, PP4419 - 44232010.

[8] Y.P. Fallah, H. Ching-Ling, R. Sengupta and Krishnan, "Analysis of Information Dissemination in Vehicular Ad-Hoc Networks With APPlication to Cooperative Vehicle Safety Systems", Vehicular Technology, IEEE Transactions on, PP 233 - 247, 2011.

[9] S. Panichpapiboon and W. Pattara-atikom, "A Review of Information Dissemination Protocols for Vehicular Ad Hoc Networks", IEEE Communications Surveys \& Tutorials, PP $784-798,2011$.

[10] N. Alam, A. Tabatabaei Balaei, A. G. Dempster, "Relative Positioning Enhancement in VANETs: A Tight Integration APProach", Intelligent Transportation Systems, IEEE Transactions on, 2012.

[11] H. Moustafa and Y. Zhang, "Vehicular Networks Techniques, Standards and Applications", Taylor and Francis Group, LLC. 2009. 
[12] J. Jansons and A. Barancevs, "Using wireless networking for vehicular environment: IEEE 802.11a standard performance", Digital Information Processing and Communications (ICDIPC), 2012 Second International Conference, PP 5 - 9, 2012.

[13] OPNET Technologies, OPNET Modeler Product Documentation Release 14.5, OPNET Modeler, 2008.

[14] M. Saito, M. Funai, T. Umedu and T. Higashino, "Inter-Vehicle Ad-Hoc Communication Protocol For Acquiring Local Traffic Information”, Osaka University, JAPAN, 2005.

[15] H. Hartenstein and K. P. Laberteaux, "VANET: Vehicular Applications and InterNetworking Technologies", John Wiley \& Sons Ltd, This edition first published, 2010.

[16] L.Tung, M. Gerla, "An efficient road-based directional broadcast protocol for urban VANETs", Vehicular Networking Conference (VNC), 2010 IEEE, PP 9 - 16, Dec. 2010,

[17] P. Gupta and P. R. Kumar, "The Capacity of Wireless Networks", IEEE Transactions on Information Theory, VOL. 46, NO. 2, PP 388 404, March 2000.

[18] "highway capacity manual 2000: Transportation Research Board; Lslf edition", Transportation Research Board of the National Academies of Science in the United States, December 2000.

[19] E. Olsen, A. B. McClurg and J. M. Bunker, "A Four Level Road Hierarchy for Network Planning and Management", In Jaeger, Vicki (Ed.) 20th ARRB Conference, PP 1-13, Nov 2005.

[20] A. P. Chen, "Vehicular Network Simulation Platform for Highway Traffic Management and Wireless", Master thesis, university of California, 2007.

[21] S. Chang, J. Jung, J. Cha and S. Lee, "Implementation of DSRC Mobile MAC for VANET", Advanced Communication Technology (ICACT), 2011 13th International Conference, PP1502 - 1505, 2011.

[22] L. Wischhof, A. Ebner, H. Rohling, M. Lott and R. Halfman, "SOTIS - A SelfOrganizing Traffic Information System", VTC '03-spring: Proceedings of the 57th IEEE Vehicular Technology Conference, PP 2442-2446, 2003.

تم اجراء البحث في كلية ألهندسة = جامعة ألموصل 